\title{
EINE DARSTELLUNG DER INTUITIONISTISCHEN LOGIK IN DER KLASSISCHEN
}

\author{
SHÔJI MAEHARA
}

Für jeden, der sich an die klassische Logik gewöhnt ist, mag undenkbar sein, dass der "Satz vom ausgeschlossenen Dritten" in der intuitionistischen Logik im allgemeinen nicht anerkannt ist. Aber es ist ganz natürlich, wenn man diejenige Tatsache beobachtet, dass eine äusserlich gleiche Aussage in der klassischen Logik und in der intuitionistischen doch ungleiche inhaltliche Sinne haben kann.

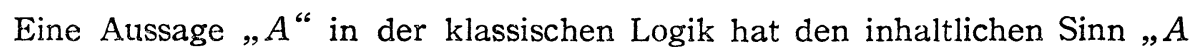
ist richtig in der Tat “_ ob man wissen kann oder nicht_-, und in der intuitionistischen Logik aber hat sie den inhaltlichen Sinn ,es ist beweisbar dass $A$ richtig ist". Die Aussage "nicht $A$ “ in der klassischen Logik bedeutet nur " $A$ ist falsch", aber in der intuitionistischen Logik bedeutet sie „es ist beweisbar dass $A$ falsch ist".

Aber wir können die obigen zwei Arten der Logik so erklären, dass sich jede Aussage in der einen Logik in eine sinnverwandte Aussage in der anderen Logik übersetzen lässt. Kuroda [2] ${ }^{11}$ hat ja jede Aussage in der klassischen Logik in eine Aussage in der intuitionistischen übersetzt. Im folgenden werden wir uns umgekehrt klar machen, dass sich jede Aussage in der intuitionistischen Logik in eine Aussage in der klassischen übersetzen lässt.

In dieser Abhandlung wollen wir die klassische und intuitionistische Logik mit dem Gentzenschen " „Kalkül $L K$ bzw. $L J$ “ und verwandten Systemen darstellen; also beziehen sich die folgenden Untersuchungen auf den Bereich der Prädikatenlogik. Im folgenden verwenden wir die Terminologie und die Zeichen, mit wenigen Ausnahmen, nach Gentzen [1].

In $\S 1$ betrachten wir den Kalkül $L J$ als die Vorbereitung zu $\S 3$.

Eingegangen am 20, Okt. 1953.

1) Siehe die Liste der Literaturen am Ende.

2) Vgl. Gentzen [1]. 
In $\S 2$ führen wir ein neues Aussagenverknüpfungszeichen „Bew “ ein, d.h. : Wenn $\mathfrak{A}$ eine Formel ist, so betrachten wir auch

Bew $\mathfrak{A}$

als eine Formel, was wir „, $\mathfrak{A}$ ist beweisbar“ lesen wollen.

Es ist natürlich, dass Bew, das in den Kalkül $L K$ eingeführt wird, bedeutet: „beweisbar in der klassischen Logik-also eventuell durch den Satz vom ausgeschlossenen Dritten___, und dass Bew, das in den Kalkül $L J$ eingeführt wird, bedeutet: „beweisbar in der intuitionistischen Logik-also ohne den Satz vom ausgeschlossenen Dritten__.".

Nunmehr nennen wir den Schlussweisenkalkül, der durch die Einführung des Verknüpfungszeichens Bew in den Kalkül $L K$ bzw. $L J$ zustande kommt, den Kalkül $B L K$ bzw. $B L J$.

In $\S 3$ beweisen wir den folgenden Satz, in dem $\mathfrak{A}$ eine Formel im Kalkül $L J$, und $\mathfrak{A}^{b}$ diejenige Formel im Kalkül $B L K$ ist, welche durch die Ersetzung jeder Teilformel $\mathfrak{I}$ von $\mathfrak{A}$ durch Bew $\mathfrak{I}$ entsteht. ${ }^{3)}$

Hauptsatz $^{4)}: \quad \mathfrak{U}$ ist dann und nur dann herleitbar im Kalkül $L J$, wenn $\mathfrak{H}^{b}$ im Kalkül $B L K$ herleitbar ist.

Nach diesem Hauptsatz dürfen wir einsehen, dass eine Aussage, d.h. eine mit der Formel $\mathfrak{I}$ dargestellte Aussage in der intuitionistischen Logik, und die mit der Formel $\mathfrak{H}^{b}$ dargestellte Aussage in der klassischen Logik beide denselben inhaltlichen Sinn haben. Der Titel dieser Abhandlung bedeutet ja nichts anderes.

Ferner soll der folgende Satz bewiesen werden.

Zusatz $^{5)}$ des Satzes 2: Sei $\mathfrak{A}$ eine Formel im Kalkül $L J$, so ist $\mathfrak{A}^{b}$ dann und nur dann herleitbar im Kalkül $B L J$, wenn $\mathfrak{A}$ im Kalkül $L J$ herleitbar ist.

Nach diesem Zusatz dürfen wir einsehen, dass der Begriff beweisbar schon in einer beliebigen Aussage in der intuitionistischen Lokig enthalten ist.

Nach den obigen zwei Sätzen ist es natürlich, dass man zum Beweis der mit der Formel der Gestalt $\mathfrak{H}^{b}$ dargestellten Aussage den Satz vom ausgeschlossenen Dritten anwenden darf, ob man seine Gültigkeit anerkennt oder nicht. Denn man kann aus dem Beweise den Satz vom ausgeschlossenen Dritten

3) Um Einzelheiten wende man sich an 3.1.

4) Vgl. 3.7.

5) Vgl. 3.51. 
weglassen, wenn es nötig ist. ${ }^{6)}$

Wegen der Bequemlichkeit zählen wir die Schlussfigurenschemata in den Kalkülen $L K$ und $L J$ anf. Um Einzelheiten wende man sich an Gentzen [1].

Schemata für Struktur-Schlussfiguren:

Verdünnung :

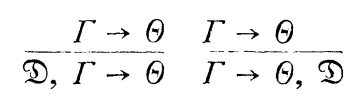

Zusammenziehung:

$\mathfrak{D}, \mathfrak{D}, \Gamma \rightarrow \Theta \quad \Gamma \rightarrow \Theta, \mathfrak{D}, \mathfrak{D}$

$$
\mathscr{D}, \Gamma \rightarrow \Theta \quad \Gamma \rightarrow \Theta, D
$$

Vertauschung :

$\Delta, \mathfrak{D},(\mathfrak{E}, \Gamma \rightarrow \Theta \quad \Gamma \rightarrow \Theta, \mathfrak{E}, \mathfrak{D}, A$

Schnitt :

$$
\begin{gathered}
\Delta, \mathfrak{D}, \mathbb{D}, \Gamma \rightarrow \Theta \quad \Gamma \rightarrow \Theta, \mathcal{D}, \mathcal{E}, A \\
\Gamma \rightarrow \Theta, \mathcal{D} \quad \mathfrak{D}, \Delta \rightarrow A \\
\Gamma, \Delta \rightarrow \Theta, A
\end{gathered}
$$

Schemata für Verknüpfungs-Schlussfiguren:

UES: $\frac{\Gamma \rightarrow \Theta, \mathfrak{H} \Gamma \rightarrow \Theta, \mathfrak{B}}{\Gamma \rightarrow \Theta, \mathfrak{A} \wedge \mathfrak{B}}$

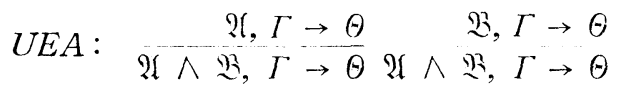

AES: $\frac{\Gamma \rightarrow \Theta \cdot \pi(a)}{\Gamma \rightarrow \Theta, \forall \underline{r} \text { 市 }(\underline{x})}$

$A E A: \quad \begin{aligned} \tilde{y}(\mathrm{t}), I & \rightarrow \Theta \\ \forall \mathrm{x}(\mathrm{r}), I & \rightarrow \Theta\end{aligned}$

NES: $\begin{aligned} \quad \mathfrak{Y}, I & \rightarrow \theta \\ I & \rightarrow \theta, \rightarrow \mathfrak{A}\end{aligned}$

FES : $\quad \frac{\mathfrak{N}, \Gamma \rightarrow \theta, \mathfrak{2}}{\Gamma \rightarrow \theta, \mathfrak{A}+\mathfrak{B}}$
OEA $: \frac{\mathfrak{R}, \Gamma \rightarrow \Theta \frac{\mathfrak{R}, \Gamma \rightarrow \Theta}{\mathfrak{A} \vee \mathfrak{B}, \Gamma \rightarrow \Theta}}{\Gamma \rightarrow \Theta}$

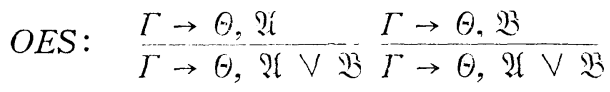

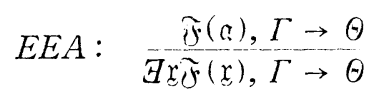

EES: $\quad \Gamma \rightarrow \Theta, \delta(\mathrm{t})$

$\Gamma \rightarrow \Theta, \exists \mathrm{Br} \widetilde{S}(\mathrm{x})$

NEA: $\quad \begin{aligned} \Gamma \rightarrow \Theta, \mathfrak{H} \\ \quad \mathfrak{A}, \Gamma \rightarrow \Theta\end{aligned}$

FEA : $\frac{\Gamma \rightarrow \Theta, \mathfrak{A} \quad \mathfrak{R}, \Delta \rightarrow \Lambda}{\mathfrak{A} \vdash \mathfrak{B}, \Gamma, \Delta \rightarrow \Theta, A}$

Für $\mathfrak{A}, \mathfrak{B}, \mathfrak{D},(5$ darf eine beliebige Formel eingesetzt werden; für $\forall \mathfrak{x} \mathfrak{F}(\mathfrak{x})$ bzw. $\exists \mathfrak{z} \mathfrak{x}(\mathfrak{x})$ eine beliebige Formel von dieser Gestalt, alsdann ist für $\mathfrak{F}(\mathfrak{a})$ bzw. $\widetilde{\jmath}(t)$ diejenige Formel zu setzen, die aus $\mathfrak{F}(\mathfrak{x})$ entsteht, wenn man die gebundene Variable $x$ durch eine beliebige freie Variable $a$ bzw. einen beliebigen Term $t$ ersetzt. Für $\Gamma, \Delta, \Theta$ und .1 dürfen beliebige, evtl. auch leere Reihen von Formeln, durch Kommata getrennt, eingesetzt werden.

Variablenbedingung: Die mit a bezeichnete freie Variable darf in der Untersequenz dieser Schlussfigur nicht vorkommen.

Ferner gibt es für LJ-Schlussfiguren folgende Einschränkung:

6) Vgl. 3.6. Satz 3 . 
„Im Sukzedens jeder Herleitungs-Sequenz darf nicht mehr als eine Sequenzformel vorkommen."

\section{§ 1. Eine Vorbetrachtung über Kalkül $L J$.}

Wir führen einen Hilfskalkül — wollen wir Kalkül L'J nennen-ein, der sich von dem Kalkül $L K$ in folgender Weise unterscheidet.

Die Schlussfiguren dürfen nach den Schlussfigurenschemata gebildet werden, jedoch mit folgenden Einschränkungen: bei den Schemata für FES, NES und $A E S$ darf für $\Theta$ nichts eingesetzt werden, diese Stellen bleiben also leer.

Der neue Kalkül $L^{\prime} J$ ist äquivalent mit dem Kalkül $L J$.

1.1. Der Begriff der Äquivalenz."

1.11. Wir führen den folgenden Äquivalenzbegriff $z$ wischen Sequenzen ein : Gleiche Sequenzen sind äquivalent.

Die Sequenz

$$
\Gamma \rightarrow \mathfrak{A}_{\nu}, \ldots, \mathfrak{N}_{1}
$$

ist äquivalent mit der Sequenz

$$
\Gamma \rightarrow \mathfrak{A}_{1} \vee \ldots \vee \mathfrak{U}_{\nu} \quad(\nu=1,2, \ldots) .
$$

$\left(\right.$ Mit $\mathfrak{A}_{1} \vee \mathfrak{A}_{2} \vee \ldots \vee \mathfrak{U}_{\nu}$ meinen wir $\left.\left(\left(\mathfrak{U}_{1} \vee \mathfrak{P}_{2}\right) \vee \ldots\right) \vee \mathfrak{U}_{\nu}.\right)$

1.12. Zwei Herleitungen wollen wir äquivalent nennen, wenn dic Endsequenz der einen mit der der anderen äquivalent ist。

1.13. Zwei Kalküle nennen wir äquivalent, wenn sich jede Herleitung in dem einen Kalkül in eine äquivalente Herleitung in dem anderen umwandeln lässt.

1.2. Der Äquivalenzbeweis der Kalküle LJ und L'J.

1.21. Umwandlung einer L'J-Herleitung in eine äquivalente LJ-Herleitung.

1.211. Diese geht so vor sich: Man ersetzt zunächst jede HerleitungsSequenz $\Gamma \rightarrow \Theta$ durch eine Sequenz $\Gamma \rightarrow \Theta^{*}$. Wenn $\Theta$ nicht leer ist, bezeichne $\Theta^{*}$ die zu $\Theta$ gehörigen Formeln in umgekehrter Reihenfolge und sei durch $\vee$ verbunden. Wenn $\Theta$ leer ist, sei $\Theta^{*}$ leer.

1.212. Nun haben wir bereits ein stammbaumförmiges System von Sequenzen. Die Endsequenz ist offenbar schon äquivalent mit der Endsequenz

7) Gentzen [1], V. Abschnitt, $\$ 1$. 
der $L^{\prime} J$-Herleitung. Die obersten Sequenzen haben sämtlich die Gestalt $\mathscr{D} \rightarrow \mathscr{D}$, sind also bereits Grundsequenzen einer $L J$-Herleitung.

Die aus den $L^{\prime} J$-Schlussfiguren entstandenen Figuren werden nach den folgenden Schemata im Teile einer $L J$-Herleitung umgewandelt.

1.212.1. Die Schlussfiguren: Verdünnung im Antezedens, Zusammenziehung im Antezedens, Vertauschung im Antezedens, und zwar UEA, OEA, AEA, $E E A, F E S, N E S$ und $A E S$ sind bereits durch die Ersetzung in $L J$-Schlussfiguren übergegangen. (Im Falle einer $L^{\prime} J$-AES bzw. $-E E A$ ist die $L J$-Variablenbedingung auf Grund der $L^{\prime} J$-Variablenbedingung erfüllt.)

1.212.2. Ein Schnitt wird $z u$ :

$$
\frac{\Gamma \rightarrow \mathscr{D} \vee \Theta^{*} \mathfrak{D}, \Delta \rightarrow A^{*}}{\Gamma, \Delta \rightarrow A^{*} \vee \Theta^{*}}
$$

(Wenn $\Theta$ und $A \mathfrak{A}_{\mu}, \ldots, \mathfrak{H}_{1}$ bzw. $\mathfrak{B}_{\nu}, \ldots, \mathfrak{B}_{1}$ sind, so bezeichne $A^{*} \vee \Theta^{*}$ $\mathfrak{B}_{1} \vee \ldots \vee \mathfrak{B}_{\nu} \vee \mathfrak{A}_{1} \vee \ldots \vee \mathfrak{H}_{\mu}$. Wenn $A$ leer ist, so bedeute $A^{*} \vee \Theta^{*} \Theta^{*}$. Wenn $\Theta$ leer ist, so bedente $\Lambda^{*} \vee \Theta^{*} A^{*}$.)

Ist $\Theta$ leer, so ist die Schlussfigur $L J$-Schnitt.

Sei nun $\Theta$ nicht leer: $\mathfrak{A}_{\mu}, \ldots, \mathfrak{A}_{1}$, macht man daraus :

$\mathfrak{D}, \Delta \rightarrow A^{*} \quad \frac{\mathfrak{A}_{1} \rightarrow \mathfrak{A}_{1}}{\mathfrak{A}_{1}, \Delta \rightarrow \mathfrak{H}_{1}}$ evtl. mehrmalige Verdünnungen $\mathrm{u}$. Vertauschungen

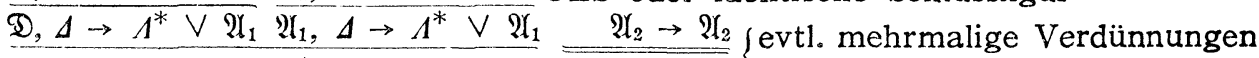

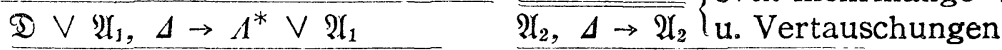

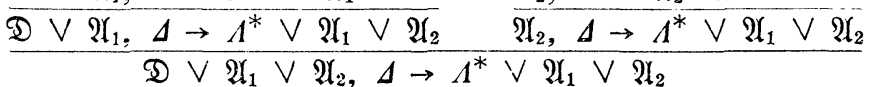

$$
\frac{\Gamma \rightarrow \mathscr{D} \vee \Theta^{*} \mathfrak{D} \vee \Theta^{*}, \Delta \rightarrow \Lambda^{*} \vee \Theta^{*}}{\Gamma, \Delta \rightarrow \Lambda^{*} \vee \Theta^{*}} L J \text {-Schnitt. }
$$

1.212.3. Eine Verdünnung im Sukzedens wird $z \mathrm{u}$ :

$$
\underset{\Gamma \rightarrow \Theta^{*}}{\Gamma \rightarrow \Theta^{*}}
$$

Ist $\Theta$ leer, so ist die Schlussfigur $L J$-Verdünnung im Sukzedens.

Sei nun $\Theta$ nicht leer: $\mathfrak{A}_{\nu}, \ldots, \mathfrak{A}_{1}$, macht man daraus : 


$$
\frac{\Gamma \rightarrow \mathfrak{T}_{1} \vee \ldots \vee \mathfrak{A}_{2} \frac{\mathfrak{A}_{1} \rightarrow \mathfrak{H}_{1}}{\mathfrak{H}_{1} \rightarrow \mathfrak{D} \vee \mathfrak{A}_{1}}}{\Gamma \rightarrow \mathfrak{D} \vee \mathfrak{A}_{1} \vee \ldots \vee \mathfrak{A}_{\nu}}(1.212 .2)^{8 !}
$$

1.212.4. Eine Zusammenziehung im Sukzedens wird zu:

$$
\frac{\Gamma \rightarrow \mathfrak{D} \vee \mathfrak{D} \vee \Theta^{*}}{\Gamma \rightarrow \mathfrak{D} \vee \Theta^{*}}
$$

Man macht daraus :

$$
\frac{\Gamma \rightarrow \mathscr{D} \vee \mathfrak{D} \vee \Theta^{*} \frac{\mathfrak{D} \rightarrow \mathscr{D} \mathscr{D} \rightarrow \mathscr{D}}{\mathfrak{D} \vee \mathfrak{D} \rightarrow \mathscr{D}}}{\Gamma \rightarrow \mathfrak{D} \vee \Theta^{*}}(1.212 .2)
$$

1.212.5. Eine Vertauschung im Sukzedens wird $z u$ :

$$
\begin{aligned}
& \Gamma \rightarrow A^{*} \vee \mathfrak{D} \vee\left(E \vee \Theta^{*}\right. \\
& \Gamma \rightarrow \Lambda^{*} \vee \mathfrak{E} \vee \mathfrak{D} \vee \Theta^{*}
\end{aligned}
$$

Man macht daraus:

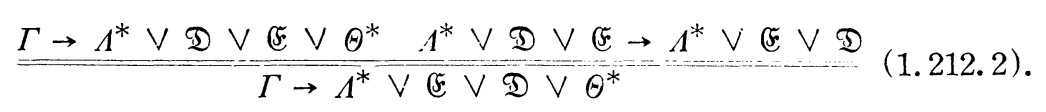

Dabei ist über $A^{*} \vee \mathfrak{D} \vee \mathfrak{F} \rightarrow A^{*} \vee \mathfrak{F} \vee \mathfrak{D}$ die in folgenden $1.212 .51 \mathrm{bzw}$. 1.212. 52 angegebene Herleitung für diese Sequenz zu setzen.

1.212.51. Wenn $\Lambda$ leer ist, die Herleitung :

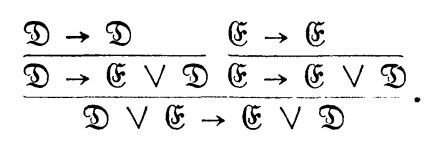

1.212.52. Wenn $A$ nicht leer ist, die Herleitung:

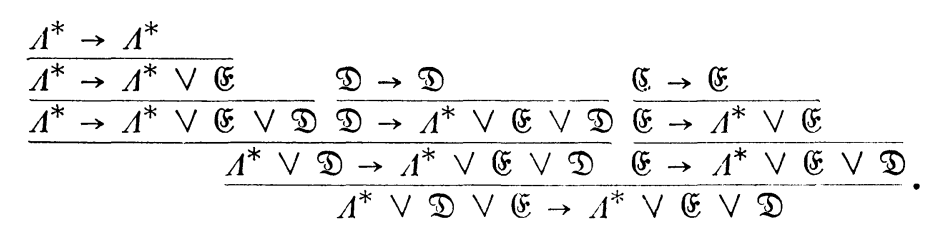

1.212.6. Eine UES wird zu:

$$
\frac{\Gamma \rightarrow \mathfrak{A} \vee \Theta^{*} \Gamma \rightarrow \mathfrak{B} \vee \Theta^{*}}{\Gamma \rightarrow(\mathfrak{A} \wedge \mathfrak{B}) \vee \Theta^{*}}
$$

8) Dies meint die Umwandlung, die in 1.212 .2 erwähnt ist. 
Man macht daraus:

$$
\begin{aligned}
& \mathfrak{U} \rightarrow \mathfrak{A} \\
& \mathfrak{B}, \mathfrak{A} \rightarrow \mathfrak{A} \quad \mathfrak{B} \rightarrow \mathfrak{B} \\
& \mathfrak{Y}, \mathfrak{B} \rightarrow \mathfrak{H} \mathfrak{A}, \mathfrak{B} \rightarrow \mathfrak{B} \\
& \Gamma \rightarrow \mathfrak{A} \vee \Theta^{*} \quad \mathfrak{A}, \mathfrak{B} \rightarrow \mathfrak{U} \wedge \mathfrak{B} \text { (1.212.2) } \\
& \Gamma, \mathfrak{B} \rightarrow(\mathfrak{A} \wedge \mathfrak{B}) \vee \Theta^{*} \text { Vertauschungen, soweit nötig } \\
& \begin{aligned}
\Gamma \rightarrow \mathfrak{B} \vee \Theta^{*} \mathfrak{B}, \Gamma \rightarrow(\mathfrak{A} \wedge \mathfrak{B}) \vee \Theta^{*} & \text { Vertauschungen, soweit nötig } \\
\frac{\Gamma, \Gamma \rightarrow(\mathfrak{A} \wedge \mathfrak{B}) \vee \Theta^{*} \vee \Theta^{*}}{\Gamma \rightarrow(\mathfrak{H} \wedge \mathfrak{B}) \vee \Theta^{*}} & \begin{array}{l}
\text { Zusammenziehungen, Vertauschungen, } \\
(1.212 .4) \text { u. (1.212.5), soweit nötig. }
\end{array}
\end{aligned}
\end{aligned}
$$

1.212.7. Eine OES wird zu:

$$
\frac{\Gamma \rightarrow \mathfrak{A} \vee \Theta^{*}}{\Gamma \rightarrow \mathfrak{A} \vee \mathfrak{B} \vee \Theta^{*}}
$$

(bzw. entsprechend für die andere Form von $O E S$, Erledigung ganz analog).

Man macht daraus :

$$
\frac{\Gamma \rightarrow \mathfrak{H} \vee \Theta^{*} \frac{\mathfrak{H} \rightarrow \mathfrak{H}}{\mathfrak{H} \rightarrow \mathfrak{H} \vee \mathfrak{B}}}{\Gamma \rightarrow \mathfrak{H} \vee \mathfrak{B} \vee \Theta^{*}}(1.212 .2)
$$

1.212.8. Eine EES wird $z u$ :

$$
\begin{aligned}
& \Gamma \rightarrow \mathfrak{W}(t) \vee \Theta^{*} \\
& \Gamma \rightarrow \mathcal{H} \mathfrak{x}(\mathfrak{x}) \vee \Theta^{*}
\end{aligned}
$$

Man macht daraus:

$$
\begin{aligned}
& \tilde{F}(t) \rightarrow \tilde{F}(t)
\end{aligned}
$$

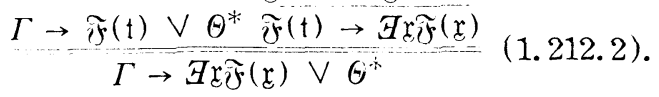

1.212.9. Eine $N E A$ wird $z u$ :

$$
\begin{aligned}
\Gamma \rightarrow \mathfrak{A} \vee \Theta^{*} \\
\neg \mathfrak{A}, \Gamma \rightarrow \Theta^{*}
\end{aligned}
$$

Man macht daraus:

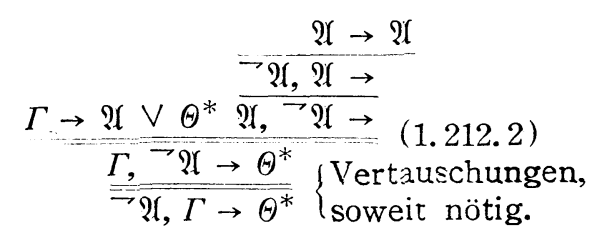


1.212.10. Eine $F E A$ wird $z \mathrm{u}$ :

$$
\frac{\Gamma \rightarrow \mathfrak{A} \vee \Theta^{*} \mathfrak{B}, \Delta \rightarrow A^{*}}{\mathfrak{A} \vdash \mathfrak{B}, \Gamma, \Delta \rightarrow A^{*} \vee \Theta^{*}}
$$

Man macht daraus :

$$
\begin{aligned}
& \frac{\mathfrak{U} \rightarrow \mathfrak{U} \quad \mathfrak{B}, \Delta \rightarrow A^{*}}{\mathfrak{U} \vdash \mathfrak{B}, \mathfrak{Y}, \Delta \rightarrow \Lambda^{*}} \\
& \frac{\Gamma \rightarrow \mathfrak{A} \vee \Theta^{*} \frac{\mathfrak{A}, \mathfrak{A} \vdash \mathfrak{B}, \Delta \rightarrow A^{*}}{\Gamma, \mathfrak{A} \vdash \mathfrak{B}, \Delta \rightarrow A^{*} \vee \Theta^{*}}}{\mathfrak{\mathscr { Y } \vdash \mathfrak { B } , \Gamma , \Delta \rightarrow A ^ { * } \vee \Theta ^ { * }}}\left\{\begin{array}{l}
\text { Vertauschungen, } \\
\text { soweit nötig. }
\end{array}\right.
\end{aligned}
$$

Damit ist die Umwandlung einer $L^{\prime} J$-Herleitung in eine äquivalente $L J$ Herleitung vollzogen.

1.22. Umwandlung einer LJ-Herleitung in eine äquivalente L'J-Herleitung.

Eine $L J$-Herleitung ist eine mit sich selbst äquivalente $L^{\prime} J$-Herleitung, also ist keine Umwandlung notwendig.

Nimmt man die Ergebnisse der 1.21 und 1.22 zusammen, so ist nunmehr die Äquivalenz der Kalküle $L J$ und $L^{\prime} J$ völlig bewiesen.

1.3. Eine Bemerkung zu der Äquivalenz der L'J-Sequenzen.

Wie wir in 1.11 schon gesehen haben, dürfen zwei verschiedene $L^{\prime} J$ Sequenzen äquivalent sein. In diesem Falle aber sind sie deduktionsgleich.

1. 31. Ist $\Gamma \rightarrow \mathfrak{A}_{1} \vee \ldots \vee \mathfrak{U}_{\nu} L^{\prime} J$-herleitbar, so ist $r \rightarrow \mathfrak{A}_{\nu}, \ldots, \mathfrak{H}_{1}$ folgendermassen $L^{\prime} J$-herleitbar:

$$
\frac{\Gamma \rightarrow \mathfrak{A}_{1} \vee \ldots \vee \mathfrak{H}_{\nu} \mathfrak{H}_{1} \vee \ldots \vee \mathfrak{P}_{\nu} \rightarrow \mathfrak{A}_{\nu}, \ldots, \mathfrak{P}_{1}}{\Gamma \rightarrow \mathfrak{U}_{v}, \ldots, \mathfrak{A}_{1}}
$$

Dabei ist die Sequenz $\mathfrak{A}_{1} \vee \ldots \vee \mathfrak{A}_{\nu} \rightarrow \mathfrak{A}_{\nu}, \ldots, \mathfrak{H}_{1} L^{\prime} J$-herleitbar.

1.32. Ist $\Gamma \rightarrow \mathfrak{A}_{\nu}, \ldots, \mathfrak{A}_{1} L^{\prime} J$-herleitbar, so ist $\Gamma \rightarrow \mathfrak{U}_{1} \vee \ldots \vee \mathfrak{A}_{\nu} \quad L^{\prime} J$ herleitbar (vgl. 1.21 und 1.22).

1.4. In den folgenden Untersuchungen meinen wir mit dem Kalkül $L J$ den Kalkül $L^{\prime} J$.

\section{§2. Neues Verknüpfungszeichen "Bew “.}

2.1. Wir erweitern zunächst den Begriff Formel, der sich von diesem im Kalkül $L K$ bzw. $L J$ in folgender Weise unterscheidet, d.h.:

Ist $\mathfrak{A}$ eine Formel, so ist auch Bew $\mathfrak{A}$ eine Formel.

2.2. Wir fügen folgende zwei Schemata für Schlussfiguren hinzu: 


$$
B E S^{9)} \frac{\Gamma^{\prime} \rightarrow \mathfrak{I}}{\Gamma^{\prime} \rightarrow \text { Bew } \mathfrak{I}} \quad B E A^{9)} \frac{\mathfrak{A}, \Gamma \rightarrow \Theta}{\text { Bew } \mathfrak{A}, \Gamma \rightarrow \Theta}
$$

Bei dem Schema für $B E S$, kann für $\Gamma^{\prime}$ eine beliebige (eventuell leere) Reihe von Formeln mit Bew als äusserstem Zeichen, durch Kommata getrennt, eingesetzt werden.

2.3. Den Schlussweisenkalkül, der aus dem Kalkül $L K$ bwz. $L J$ unter 2.1 und 2.2 entsteht, wollen wir Kalkül $B L K$ bzw. $B L J$ nennen.

2.4. Einige Bemerkungen zu den neuen Schlussfigurenschemata.

2.41. Im Kalkül $B L K$ bzw. $B L J$ lassen sich die Schlussfiguren $B E A$ durch Grundsequenzen nach folgendem Schema ersetzen:

$$
\text { Bew } \mathfrak{A} \rightarrow \mathfrak{H} \text {. }
$$

Die Äquivalenz dieser Grundsequenz mit der Schlussfigur $B E A$ ist durch Schnitt leicht zu zeigen.

2. 42. Im Kalkül $B L K$ bzw. $B L J$ lassen sich die Schlussfiguren $B E S$ durch Grundsequenzen und Schlussfiguren nach folgenden Schemata ersetzen:

$$
\text { Bew } \mathfrak{i} \rightarrow \text { Bew }(\text { Bew } \mathfrak{I})
$$

und

$$
\frac{\Gamma \rightarrow \mathfrak{H}}{\operatorname{Bew} T \rightarrow \operatorname{Bew} \mathfrak{U}}
$$

Wenn $\Gamma$ leer ist, sei Bew $\Gamma$ leer. Wenn $\Gamma \mathfrak{A}_{1}, \ldots, \mathfrak{A}_{v}$ ist, bezeichne Bew $\Gamma$ Bew $\mathfrak{A}_{1}, \ldots$, Bew $\mathfrak{A}_{2}(\nu=1,2, \ldots)$.

Die Äquivalenz der Grundsequenzen und Schlussfiguren mit den Schlussfiguren $B E S$ ist durch Vertauschungen im Antezedens, Schnitte und $B E A$ leicht zu zeigen.

2.5. Die Beispiele der in Kalkülen BLK und BLJ herleitbaren Sequenzen.

Jede Sequenz in der folgenden Gestalt ist in beiden Kalkülen $B L K$ und $B L J$ herleitbar.

2.511. Bew $(\mathfrak{A} \wedge \mathfrak{B}) \rightarrow$ Bew $\mathfrak{A} \wedge$ Bew $\mathfrak{B}$

2.512. Bew $\mathfrak{A} \wedge$ Bew $\mathfrak{B} \rightarrow \operatorname{Bew}(\mathfrak{A} \wedge \mathfrak{B})$

9) Die Bedeutungen dieser Bezeichnungen:

EES (,Beweisbar"-Einführung im Sukzedens)

$B E A$ ( ,Beweisbar"-Einführung im Antezedens). 
2.521. Bew (Bew $\mathfrak{A} \vee$ Bew $\mathfrak{B}) \rightarrow$ Bew $\mathfrak{i} \vee$ Bew $\mathfrak{B}$

2.522. Bew $\mathfrak{A} \vee$ Bew $\mathfrak{B} \rightarrow$ Bew (Bew $\mathfrak{A} \vee$ Bew $\mathfrak{B}$ )

2.531. Bew $(\forall \mathfrak{r}$ Bew $\mathfrak{F}(\mathfrak{x})) \rightarrow \operatorname{Bew}(\forall \mathfrak{x} \mathfrak{F}(\mathfrak{r}))$

2.532. Bew $(\forall \mathfrak{x} \mathfrak{F}(\mathfrak{x})) \rightarrow \operatorname{Bew}(\forall \mathfrak{x}$ Bew $\mathfrak{F}(\mathfrak{x}))$

2.541. Bew $(\exists \mathfrak{g}$ Bew $\widetilde{F}(\mathfrak{x})) \rightarrow \exists \mathfrak{Z}$ Bew $\widetilde{F}(\mathfrak{x})$

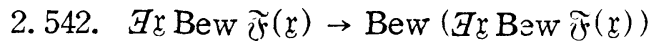

2.6. Im Kalkül $B L K$, wie im Kalkül $L K$, lautet der Satz:

Jede $B L K$-Herleitung lässt sich in eine $B L K$-Herleitung mit der gleichen Endsequenz umwandeln, in welcher die als Schnitt bezeichnete Schlussfigur nicht vorkommt.

Dieser Satz ist bewiesen, wie der Gentzensche Beweis ${ }^{10)}$ seines Hauptsatzes im Kalkül $L K$, durch die Einführung der „Mischung“ und durch zwei vollständige Induktionen nach dem Grad und dem Rang der Herleitung, die eine Mischung als unterste Schlussfigur hat, und sonst keine Mischungen enthält.

Dabei aber müssen wir folgendes hinzufügen, um den vollständigen Beweis zu bekommen.

2.61. Es sei Rang $=2$, die Mischformel $\mathfrak{M}$ komme im Sukzedens der linken und im Antezedens der rechten Obersequenz der Mischung lediglich als Hauptformel je einer Verknüpfungs-Schlussfigur vor, und zwar das äusserste Zeichen von $\mathfrak{M}$ sei Bew. ${ }^{11)}$ Dann lautet das Ende der Herleitung:

$$
\frac{\frac{\Gamma^{\prime} \rightarrow \mathfrak{A}}{\Gamma^{\prime} \rightarrow \operatorname{Bew} \mathfrak{A}} B E S \quad \begin{array}{l}
\mathfrak{A}, \Gamma \rightarrow \Theta \\
\text { Bew } \mathfrak{A}, \Gamma \rightarrow \Theta
\end{array} B E A}{\Gamma^{\prime}, \Gamma \rightarrow \Theta} \text { Mischung. }
$$

Man lässt es umwandeln in

$$
\begin{aligned}
& \frac{\Gamma^{\prime} \rightarrow \mathfrak{H} \mathfrak{A}, \Gamma \rightarrow \Theta}{\Gamma^{\prime}, \bar{\Gamma} \rightarrow \Theta} \text { Mischung } \\
& \frac{\Gamma^{\prime}, \Gamma \rightarrow \Theta}{\text { evtl. mehrmalige Verdünnungen }} \\
& \text { u. Vertauschungen. }
\end{aligned}
$$

Dabei ist die Formelreihe $\bar{\Gamma}$ von $\Gamma$, jedoch mit Weglassung sämtlicher vorkommender Formeln der Gestalt $\mathfrak{\Re}$, entstanden.

Auf dem Herleitungsteil mit der untersten Sequenz $\Gamma^{\prime}, \bar{\Gamma} \rightarrow \Theta$ lässt sich nun die Induktionsannahme in Bezug auf Grad anwenden, dann hat er einen kleineren Grad als der der alten Herleitung. Also lässt sich auch die ganze

10) Gentzen [1], III. Abschnitt, $\$ 3$.

11) Gentzen [1], III. Abschnitt, 3.113.3. 
Herleitung in eine von Mischungen freie Herleitung umwandeln.

2.62. Es sei Rang $>2$.

2.621. Die rechte Rangzahl sei grösser als 1, die Mischformel $\mathfrak{X}$ im Antezedens der linken Obersequenz der Mischung komme nicht vor und die rechte Obersequenz der Mischung sei die Untersequenz einer BES. ${ }^{12)}$ Dann lautet das Ende der Herleitung:

$$
\frac{\prod \rightarrow \Sigma \frac{\Gamma^{\prime} \rightarrow \mathfrak{H}}{\Gamma^{\prime} \rightarrow \text { Bew } \mathfrak{H}}}{I I, \overline{\Gamma^{\prime}} \rightarrow \bar{\Sigma}, \text { Bew } \mathfrak{A}} \text { Mischung. }
$$

Dabei sind die Formelreihen $\bar{\Sigma}$ und $\Gamma^{\prime}$ aus $\Sigma$ und $\Gamma^{\prime}$ entstanden, jedoch mit Weglassung sämtlicher vorkommenden Formeln der Gestalt $\mathfrak{M}$.

$\Gamma^{\prime}$ ist Reihe von Formeln mit Bew als äusserstem Zeichen, in welchen eine Formel der Gestalt $\mathfrak{M}$ jeweils mindestens einmal auftritt, also hat $\mathfrak{M}$ die Gestalt BewD.

Nun führen wir zwei einfache Hilfsbegriffe ein:

Gleiche, einander nach dem Schlussfigurenschema entsprechende Sequenzformeln in den Obersequenzen und der Untersequenz einer SchlussfigurMischung und Schnitt ausgenommen-sollen verbunden heissen.

Die Gesamtheit aller Formeln in Herleitung, welche man erhält, wenn man von einer einzelnen Formel ausgehend alle mit ihr verbundenen Formeln, dann alle mit diesen verbundenen Formeln usw. hinzunimmt, heisse ein Formelbund; wir können auch sagen: der Bund, $z u$ dem die betreffende Ausgangsformel gehört.

Wir sagen: "Eine Herleitungs-Sequenz gehört zum Bund“, wenn eine Sequenzformel der Sequenz zum Bund gehört.

„Eine Herleitungs-Verknüpfungs-Schlussfigur gehört zum Bund“", wenn die Hauptformel der Schlussfigur zum Bund gehört.

„Eine Herleitungs-Verdünnung gehört zum Bund“, wenn die Verdünnungsformel der Verdünnung zum Bund gehört.

Nun betrachten wir den zu der Mischformel Bew $\mathfrak{D}(=\mathfrak{M})$ im Sukzedens der linken Obersequenz $\Pi \rightarrow \Sigma$ der Mischung gehörigen Bund.

Die Herleitungs-Sequenz, die mindestens eine oberste Formel des Bundes enthält, ist entweder eine Untersequenz einer BES oder einer Verdünnung im

12) Gentzen [1], III. Abschnitt, 3.121.2. 
Sukzedens, die zum Bund gehört, oder eine Grundsequenz.

2.621.1. Man ersetzt zunächst jede zum Bund gehörige HerleitungsSequenz $\Delta \rightarrow A$ durch eine Sequenz $d, \overline{\Gamma^{\prime}} \rightarrow \tilde{A}$. Dabei ist $\tilde{A}$ die Formelreihe, die aus $A$ entsteht, wenn man die zum Bund gehörige Formel durch die Formel Bew $\mathfrak{A}$ ersetzt.

2.621.2. Eine zum Bund gehörige BES:

$$
\frac{\Pi_{1}^{\prime} \rightarrow \mathscr{D}}{\Pi_{1}^{\prime} \rightarrow \text { Bew } \mathscr{D}}
$$

wird zu:

$$
\frac{\Pi_{1}^{\prime} \rightarrow \mathfrak{D}}{\Pi_{1}^{\prime}, \bar{\Gamma}^{\prime}, \rightarrow \text { Bew } \mathfrak{l l}}
$$

Man lässt es umwandeln in

$$
\frac{\frac{\Pi_{1}^{\prime} \rightarrow \mathfrak{D}}{\Pi_{1}^{\prime} \rightarrow \text { Bew } \mathscr{D}} B E S \quad \Gamma^{\prime} \rightarrow \mathfrak{U}}{\quad \frac{\Pi_{1}^{\prime}, \overline{\Gamma^{\prime}} \rightarrow \mathfrak{L}}{\Pi_{1}^{\prime}, \overline{\Gamma^{\prime}} \rightarrow \text { Bew } \mathfrak{U}} \text { BES. }} \text { Mischung }
$$

(Über $\Gamma^{\prime} \rightarrow \mathfrak{A}$ schreiben wir die zugehörige Herleitung.)

Die Herleitung für die Untersequenz der neuen Mischung hat linke Rangzahl 1, und ihre rechte Rangzahl um 1 kleiner ist als die der alten Herleitung. Also lässt sich die Mischung nach Induktionsannahme wegschaffen.

2.621.3. Eine zum Bund gehörige Verdünnung im Sukzedens:

$$
\frac{\Pi_{2} \rightarrow \Sigma_{2}}{I_{2} \rightarrow \Sigma_{2}, \text { Bew } \mathfrak{D}}
$$

wird zu:

$$
\frac{\Pi_{2},\left(\overline{\Gamma^{\prime}}\right) \rightarrow \widetilde{\Sigma_{2}}}{\Pi_{2}, \overline{\Gamma^{\prime}} \rightarrow \widetilde{\Sigma_{2}}, \text { Bew } \because}
$$

$\left(\left(\overline{\Gamma^{\prime}}\right)\right.$ bedeute $\overline{\Gamma^{\prime}}$ oder gar nicht, je nachdem die Herleitungs-Sequenz $\Pi_{2} \rightarrow \Sigma_{2}$ zum Bund gehört oder nicht.)

Man ersetzt es durch eventuell mehrmalige Verdünnungen und Vertauschungen, soweit nötig.

2.621.4. Eine zum Bund gehörige Grundsequenz:

$$
\text { Bew } \mathfrak{D} \rightarrow \text { Bew } \mathfrak{D}
$$

wird zu: 
Bew D, $\overline{\Gamma^{\prime}} \rightarrow$ Bew 2 .

Man macht daraus:

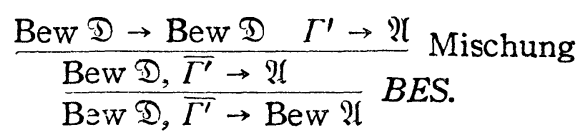

(Über $\Gamma^{\prime} \rightarrow \mathfrak{A}$ schreiben wir die zugehörige Herleitung.)

Dabei ist klar, dass in BES die in 2.2 gezeigte Bedingung erfüllt ist.

Die Herleitung für die Untersequenz der neuen Mischung hat die linke Rangzahl 1, und ihre rechte Kangzahl um 1 kleiner ist als die der alten Herleitung. Also lässt sich die Mischung nach Induktionsannahme wegschaffen.

2.621.5. Das Ende der Herleitung wird zu:

$$
\frac{I I, \overline{\Gamma^{\prime}} \rightarrow \tilde{\Sigma} \Gamma^{\prime} \rightarrow \text { Bew } \mathfrak{U}}{I, \overline{\Gamma^{\prime}} \rightarrow \bar{\Sigma}, \text { Bew } \mathfrak{U}}
$$

Man lässt es umwandeln in

$$
\frac{\Pi, \overline{\Gamma^{\prime}} \rightarrow \tilde{\Sigma}}{\overline{\Pi, \overline{\Gamma^{\prime}} \rightarrow \bar{\Sigma} \text {, Bew } \mathfrak{X}}}\left\{\begin{array}{l}
\text { evtl. mehrmalige Zusammen- } \\
\text { ziehungen u. Vertauschungen }
\end{array}\right.
$$

2.621.6. Die aus anderen Schlussfiguren entstandenen Figuren sind sämtlich bereits Schlussfiguren, die von derselben Arten wie alten Schlussfiguren sind. Es ist klar, dass in einer AES oder EEA die Variablenbedingung auf Grund der Umbenennung von freien Variablen, ${ }^{13)}$ und in einer BES, die zum Bund nicht gehört, die in 2.2 gezeigte Bedingung beide erfüllt sind.

2.622. Die rechte Rangzahl sei grösser als 1, die Mischformel im Antezedens der linken Obersequenz der Mischung komme nicht vor und die rechte Obersequenz der Mischung sei die Untersequenz einer BEA. Dieser Fall ist im wesentlichen genau so wie die anderen Verknüpfungs-Schlussfiguren zu erledigen.

2.623. Die rechte Rangzahl sei gleich 1. Dann ist die linke Rangzahl grösser als 1 .

In diesem Falle kann die Schlussfigur, welehe die linke Obersequenz der Mischung als die Untersequenz hat, nicht BES sein.

Die Schlussfigur, welehe die linke Obersequenz der Mischung als die Untersequenz hat, sei eine $B E A$. Dieser Fall ist im wesentlich genau so wie die anderen Verknüpfungs-Schlussfiguren $z u$ erledigen.

13) Gentzen [1], III. Abschnitt, 3.10. 


\section{§. Einige Sätze}

3.1. In den Kalkülen $B L K$ und $B L J$ definieren wir eine Formel $\mathfrak{A}^{b}$, was für eine beliebige Formel $\mathfrak{A}$ in folgender Weise induktiv erklärt ist.

3.11. Ist $\mathfrak{A}$ eine Primformel:

$$
\mathfrak{A}^{b}=\text { Bew } \mathfrak{A} .
$$

3. 12 .

$$
\begin{aligned}
& (\mathfrak{A} \wedge \mathfrak{B})^{b}=\operatorname{Bew}\left(\mathfrak{H}^{b} \wedge \mathfrak{B}^{b}\right) \\
& (\mathfrak{A} \vee \mathfrak{B})^{b}=\operatorname{Bew}\left(\mathfrak{H}^{b} \vee \mathfrak{B}^{b}\right) \\
& (\mathfrak{A} \vdash \mathfrak{B})^{b}=\operatorname{Bew}\left(\mathfrak{H}^{b} \vdash \mathfrak{B}^{b}\right) \\
& (\neg \mathfrak{H})^{b}=\operatorname{Bew}\left(\neg \mathfrak{H}^{b}\right) \\
& (\forall \mathfrak{x} \mathfrak{F}(\mathfrak{l}))^{b}=\operatorname{Bew}\left(\forall \mathfrak{x} \mathfrak{F}^{b}(\mathfrak{l})\right) \\
& (\exists \mathfrak{g} \mathfrak{\mathfrak { Y }}(\mathfrak{x}))^{b}=\mathrm{Bew}\left(\exists \mathfrak{x} \tilde{\mathfrak{F}}^{b}(\mathfrak{x})\right) \\
& (\text { Bew } \mathfrak{A})^{b}=\text { Bew }\left(\text { Bew } \mathfrak{H}^{b}\right)
\end{aligned}
$$

wobei $\widetilde{F}^{b}(\mathfrak{x})$ die Abbreviation von $(\mathfrak{F}(\mathfrak{x}))^{b}$ ist.

\subsection{Beispiele:}

$$
\begin{aligned}
& (A \vee \neg A)^{b}=\operatorname{Bew}(\text { Bew } A \vee \operatorname{Bew}(\neg \text { Bew } A)) \\
& (A \wedge \neg A)^{b}=\operatorname{Bew}(\text { Bew } A \wedge \operatorname{Bew}(\neg \text { Bew } A))
\end{aligned}
$$

$A$ sei eine Aussagenvariable.

3.3. Jede Sequenz in der folgenden Gestalt ist in beiden Kalkülen $B L K$ und $B L J$ herleitbar.

3.31. $\mathfrak{Q}^{b} \rightarrow$ Bew $\mathfrak{A}^{b}$

3.32. $\mathfrak{A}^{b} \wedge \mathfrak{B}^{b} \rightarrow \operatorname{Bew}\left(\mathfrak{H}^{b} \wedge \mathfrak{B}^{b}\right)$

3. 33. $\mathfrak{A}^{b} \vee \mathfrak{B}^{b} \rightarrow \operatorname{Bew}\left(\mathfrak{I}^{b} \vee \mathfrak{B}^{b}\right)$

3.34. $\exists \mathfrak{G} \tilde{\mathfrak{F}}^{b}(\mathfrak{x}) \rightarrow \operatorname{Bew}\left(\exists \mathfrak{G} \hat{\mathfrak{r}}^{b}(\mathfrak{x})\right)$

3.4. Nun bezeichnen wir mit $\Gamma^{b}$ die Formelreihe

$$
\mathfrak{A}_{1}^{b}, \mathfrak{A}_{2}^{b}, \ldots, \mathfrak{A}_{\nu}^{b},
$$

wenn $\Gamma$ die Formelreihe $\mathfrak{A}_{1}, \mathfrak{A}_{2}, \ldots, \mathfrak{A}_{\nu}$ ist $(\nu=0,1,2, \ldots)$.

Satz 1: Wenn in $\Gamma$ und $\Theta$ Verknüpfungszeichen „Bew “ nicht vorkommt und wenn

$$
\Gamma^{b} \rightarrow \Theta^{b}
$$

$B L K$ - bzw. $B L J$-herleitbar ist, dann ist 


$$
\Gamma \rightarrow \Theta
$$

$L K$ - bzw. LJ-herleitbar.

\subsection{Beweis des Satzes 1.}

3.411. Wir definieren zunächst den Begriff „Beseitigung des Verknüpfungszeichens Bew aus einer beliebigen Formel "in folgender Weise (induktiv erklärt). Die hierdurch aus Formel $\mathfrak{A}$ entstehende Formel bezeichnen wir mit $\overline{\mathfrak{A}}$.

3.411.1. Ist $\mathfrak{i}$ eine Primformel:

3. 411.2 .

$$
\begin{aligned}
& \overline{\mathfrak{I}}=\mathfrak{2} . \\
& \overline{\mathfrak{A}} \wedge \overline{\mathfrak{B}}=\overline{\mathfrak{T}} \wedge \overline{\mathbb{B}} \\
& \mathfrak{\mathfrak { A }} \vee \mathfrak{B}=\overline{\mathfrak{U}} \vee \overline{\mathfrak{B}} \\
& \mathfrak{U} \vdash \mathfrak{B}=\overline{\mathfrak{A}} \vdash \overline{\mathfrak{B}} \\
& \overline{\mathfrak{U}}=-\overline{\mathfrak{U}} \\
& \overline{\forall \mathfrak{x} \tilde{F}(x)}=\forall x \widetilde{f}(\mathfrak{x}) \\
& \overline{\text { GxF }}(\mathfrak{x})=\overline{G x} \widetilde{F}(\mathfrak{x}) \\
& \overline{\text { Bew } \mathfrak{U}}=\overline{\mathfrak{A}}
\end{aligned}
$$

3. 412. Man ändert alle Herleitungs-Sequenzen der $B L K$ - bzw. $B L J$-Herleitung, die $\Gamma^{b} \rightarrow \Theta^{b}$ als Endsequenz hat, so ab:

Aus

$$
\begin{aligned}
& \mathfrak{T}_{1}, \ldots, \mathfrak{P}_{\mu} \rightarrow \mathfrak{B}_{\nu}, \ldots, \mathfrak{B}_{1} \\
& \overline{\mathfrak{A}}_{1}, \ldots, \overline{\mathfrak{H}}_{\mu} \rightarrow \overline{\mathfrak{B}}_{\nu}, \ldots, \overline{\mathfrak{B}}_{1} .
\end{aligned}
$$

wird

Nun haben wir bereits ein stammbaumförmiges System von $L K$ - bzw. $L J$ Sequenzen. Die Endsequenz ist offenbar $I \rightarrow \Theta$. Die obersten Sequenzen haben sämtlich die Gestalt $\bar{D} \rightarrow \overline{\mathcal{D}}$, sind also bereits Grundsequenzen einer $L K-\mathrm{bzw}$. $L J$-Herleitung. Die aus den $B L K$ - bzw. $B L J$-Schlussfiguren entstandenen Figuren sind sämtlich bereits $L K$ - bzw. $L J$-Schlussfiguren, die von der gleichen Art wie $B L K$ - bzw. $B L J$-Schlussfiguren sind, aber die eventuell vorhandenen aus $B E S$ oder $B E A$ entstandenen Figuren sind die identischen Schlussfiguren.

Dann ist das stammbaumförmiges System eine $L K$ - bzw. $L J$-Herleitung, die $\Gamma \rightarrow \Theta$ als Endsequenz hat. Damit ist der Satz 1 bewiesen.

\subsection{Satz 2: Wenn}

$$
\Gamma \rightarrow \Theta
$$

$L J$-herleitbar ist, dann ist 


$$
\Gamma^{b} \rightarrow \Theta^{b}
$$

$B L J$-herleitbar。

3.51. Zusatz: Kommt das Verknüpfungszeichen „Bew “ in $\Gamma$ und $\Theta$ nicht vor, so ist

$$
\Gamma^{b} \rightarrow \Theta^{b}
$$

dann und nur dann $B L J$-herleitbar, wenn

$$
\Gamma \rightarrow \Theta
$$

$L J$-herleitbar ist.

3.511. Inhaltlich zeigt dieser Zusatz, dass das Aussagenverknüpfungszeichen „Bew “ in der intuitionistischen Logik unnötig ist.

3.512. Auf Grund der Sätze 1 und 2 ist dieser Zusatz klar.

3.52. Beweis des Satzes 2.

Es sei nun $L J$-Herleitung, die $\Gamma \rightarrow \Theta$ als die Endsequenz hat, vorhanden:

3.521. So gibt es eine $L J$-Herleitung, die $\Gamma \rightarrow \Theta^{*}$ (vgl. 1.211) als Endsequenz hat, und jede zugehörige Herleitungs-Sequenz in ihrem Sukzedens nicht mehr als eine Formel hat (vgl. 1.21).

Bezeichnen wir diese $L J$-Herleitung mit $H$.

3.522. Man ersetzt jede Herleitungs-Sequenz $\Delta \rightarrow A$ von $H$ durch $\Delta^{b} \rightarrow A^{b}$.

Nun haben wir bereits ein stammbaumförmiges System von $B L J$-Sequenzen. Die Endsequenz ist offenbar $\Gamma^{b} \rightarrow \Theta^{* b}$. Die obersten Sequenzen haben sämtlich die Gestalt $\mathfrak{D}^{b} \rightarrow \mathfrak{D}^{b}$, sind also bereits Grundsequenzen einer $B L J$-Herleitung. Die aus den $L J$-Schlussfiguren von $H$ entstandenen Figuren werden nach den folgenden Schemata in Teile ener $B L J$-Herleitung umgewandelt.

3.522.1. Jede $L J$-Struktur-Schlussfigur von $H$ ist bereits durch die obige Ersetzung in eine $B L J$-Struktur-Schlussfigur übergegangen.

3.522.21. Eine $A E S$ von $H$ :

$$
\frac{\Delta \rightarrow \tilde{J}(\mathfrak{a})}{\Delta \rightarrow \forall \mathfrak{x} \mathfrak{F}(\mathfrak{x})}
$$

wird zu :

$$
\frac{\Delta^{b} \rightarrow \mathfrak{f}^{b}(a)}{A^{b} \rightarrow \operatorname{Bew}\left(\operatorname{Vx} \mathfrak{\mho}^{b}(\mathfrak{x})\right)}
$$

Man macht daraus: 


$$
\frac{\frac{\Delta^{b} \rightarrow \widetilde{\mho}^{b}(\mathfrak{a})}{\Delta^{b} \rightarrow \forall \mathfrak{x} \tilde{\mho}^{b}(\mathfrak{x})} A E S}{\Delta^{b} \rightarrow \operatorname{Bew}\left(\forall \mathfrak{x} \hat{\mho}^{b}(\mathfrak{x})\right)} \text { BES. }
$$

Dabei ist klar, dass in dieser AES die Variablenbedingung, und in $B E S$ die in 2.2 gezeigte Bedingung beide erfüllt sind (vgl. 3.1).

3.522.22. Eine $A E A$ von $H$ :

$$
\frac{\tilde{F}(\mathrm{t}), \Delta \rightarrow A}{\forall \mathfrak{V} \mathfrak{F}(\mathfrak{x}), \Delta \rightarrow A}
$$

wird zu :

$$
\frac{\tilde{f}^{b}(\mathfrak{t}), \Delta^{b} \rightarrow \Lambda^{b}}{\operatorname{Bew}\left(\forall \mathfrak{x} \tilde{F}^{b}(\mathfrak{x})\right), \Delta^{b} \rightarrow \Lambda^{b}} .
$$

Man macht daraus :

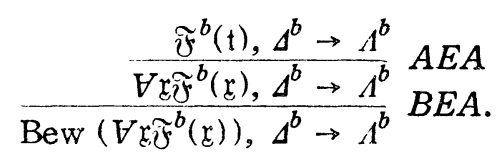

3.522.23. Für die anderen Verknüpfungszeichen $\wedge, \vee, \vdash, \rightarrow$ und $\exists$ ist die Umwandlung ganz analog.

Durch die obige Umwandlung haben wir eine $B L J$-Herleitung, die $\Gamma^{b} \rightarrow \Theta^{* b}$ als die Endsequenz hat, und also natürlich ist $\Gamma^{b} \rightarrow \Theta^{* b} B L J$-herleitbar.

3.523. Ist $\Gamma^{b} \rightarrow \Theta^{* b} B L J$-herleitbar, so ist auch $\Gamma^{b} \rightarrow \Theta^{b} B L J$-herletbar.

3.523.1. Diese Tatsache ist klar, wenn $\Theta$ leer ist.

3.523.2. Wenn $\Theta$ nicht leer ist:

Um diese Tatsache $z u$ beweisen, hat man nur zu zeigen dass die Sequenz:

$$
\Theta^{* b} \rightarrow \Theta^{b}
$$

$B L J$-herleitbar ist.

Wenn $\Theta \mathfrak{A}_{\nu}, \ldots, \mathfrak{A}_{2}, \mathfrak{A}_{1}(\nu=1,2, \ldots)$ ist, so ist die Sequenz $\Theta^{* b} \rightarrow \Theta^{b}$

$$
\operatorname{Bew}\left(\operatorname{Bew}\left(\operatorname{Bew}\left(\mathfrak{A}_{1}^{b} \vee \mathfrak{A}_{2}^{b}\right) \vee \ldots\right) \vee \mathfrak{A}_{\nu}^{b}\right) \rightarrow \mathfrak{P l}_{\nu}^{b}, \ldots, \mathfrak{I}_{2}^{b}, \mathfrak{H}_{1}^{b} .
$$

Diese Sequenz wird, durch eventuell mehrmalige $O E A, B E A$ und Verdünnungen im Sukzedens, im Kalkül $B L J$ herleitet.

Damit ist der Satz 2 vollständig bewiesen.

3.6. Satz 3: Ist die Sequenz 


$$
\Gamma^{b} \rightarrow \Theta^{b}
$$

$B L K$-herleitbar, so ist sie auch $B L J$-herleitbar.

3.61. Beweis des Satzes 3.

Wenn $\Gamma^{b} \rightarrow \Theta^{b} B L K$-herleitbar ist, so gibt es eine $B L K$-Herleitung, welche die Sequenz $\Gamma^{b} \rightarrow \Theta^{b}$ als die Endsequenz hat, und in welcher die als Schnitt bezeichnete Schlussfigur nicht vorkommt (vgl. 2.6).

Als Grad der BLK-Herleitung ohne Schnitt bezeichnen wir die Anzahl der Herleitungs-Schlussfiguren AES, NES und FES, in welchen die Untersequenz mindestens zwei Formeln in ihrem Sukzedens enthalten.

Der Grad einer Herleitung ist Null oder eine natürliche Zahl.

Zum Beweis dieses Satzes machen wir die vollständige Induktion nach dem Grad der $B L K$-Herleitung, die keinen Schnitt hat, d.h.:

3.611. Im Falle Grad $=0$, ist die $B L K$-Herleitung eine $B L J$-Herleitung, und also ist $\Gamma^{b} \rightarrow \Theta^{b} B L J$-herleitbar.

3.612. Im Falle Grad $>0$ gibt es mindestens eine Schlussfigur AES, NES oder FES, in welcher die Untersequenz mindestens zwei Formeln in ihrem Sukzedens enthält. Nun betrachten wir den Formelbund, zu dem die Hauptformel $\mathfrak{S}$ dieser Schlussfigur gehört (vgl. 2.621).

Die Herleitungs-Sequenz, die mindestens eine oberste Formel des Bundes enthält, ist entweder eine Grundsequenz oder eine Untersequenz einer Schlussfigur, die zum Bund gehört (vgl. 2.621). Die Grundsequenz, die Verdünnung und die Verknüpfungs-Schlussfigur, die zum Bund gehören, haben folgende Gestalten:

$$
\mathscr{S} \rightarrow \mathscr{S} \quad \frac{\Gamma_{1} \rightarrow \Theta_{1}}{\Gamma_{1} \rightarrow \Theta_{1}, \mathfrak{S}} \quad \frac{\Psi, \Gamma_{2} \rightarrow \Theta_{2}, \Omega}{\Gamma_{2} \rightarrow \Theta_{2}, \mathfrak{g}}
$$

Dabei sind $\Psi$ und $\Omega$ entweder Nebenformel der Schlussfigur oder leer, und also sind sie nur von der Hauptformel $\mathfrak{S}$ abhängig. (Mindestens eine der zum Bund gehörigen Verknüpfungs-Schlussfiguren hat mindestens zwei Formeln im Sukzedens der Untersequenz.)

Die Endsequenz der Herleitung ist $\Gamma^{b} \rightarrow \Theta^{b}$, und die Herleitung hat keinen Schnitt, also ist die Herleitungs-Sequenz, die die unterste Formel des Bundes enthält, eine Obersequenz einer BES_-wollen wir sie Endschlussfigur des Bundes nennen-.

3.612.1. Man ersetzt zunächst jede Herleitungs-Sequenz $\Delta \rightarrow A$, die zum 
Bund gehört, durch eine Sequenz $\Delta, \Psi \rightarrow \Omega, \tilde{\Lambda}$. Dabei ist die Formelreihe $\widetilde{\Lambda}$ aus $A$ entstanden, jedoch mit Weglassung sämtlicher vorkommenden Formeln, die zum Bund gehören.

3.612.2. Eine zun Bund gehörige Grundsequenz wird zu:

$$
\mathfrak{S}, \Psi \rightarrow \Omega .
$$

Das äusserste Zeichen der $\mathfrak{S}$ ist $\forall, \rightarrow$ oder $\vdash$, also hat die neue Sequenz die Gestalt:

$$
\forall \mathfrak{E} \mathfrak{F}(\mathfrak{x}) \rightarrow \mathfrak{F}(\mathfrak{a}) \quad \neg \mathfrak{A}, \mathfrak{H} \rightarrow \quad \text { oder } \quad \mathfrak{H} \vdash \mathfrak{B}, \mathfrak{A} \rightarrow \mathfrak{B} .
$$

Dann ist diese Sequenz $B L J$-herleitbar.

Über $\mathscr{S}, \Psi \rightarrow \Omega$ schreibt man die zugehörige $B L J$-Herleitung.

3.612.3. Eine zum Bund gehörige Verdünnung wird zu:

$$
\begin{aligned}
\Gamma_{1},(\Psi) \rightarrow(\Omega), \widetilde{\Theta}_{1} \\
\Gamma_{1}, \Psi \rightarrow \Omega, \widetilde{\Theta}_{1}
\end{aligned}
$$

( $(\Psi)$ und $(\Omega)$ bedeuten $\Psi$ und $\Omega$ oder gar nicht, je nachdem die HerleitungsSequenz $\Gamma_{1} \rightarrow \Theta_{1}$ zum Bund gehört oder nicht.)

Man ersetzt es durch eventuell mehrmalige Verdünnungen und Vertauschungen, soweit nötig.

3.612.4. Eine zum Bund gehörige Verknüpfungs-Schlussfigur wird zu:

$$
\begin{aligned}
& \Psi, \Gamma_{2},(\Psi) \rightarrow(\Omega), \widetilde{\Theta}_{2}, \Omega \\
& \Gamma_{2}, \Psi \rightarrow \Omega, \widetilde{\Theta}_{2}
\end{aligned}
$$

Man ersetzt es durch eventuell mehrmalige Zusammenziehungen und Vertauschungen, soweit nötig.

3.612.5. Die Endschlussfigur des Bundes:

$$
\frac{\Gamma_{3}^{\prime} \rightarrow \mathscr{S}}{\Gamma_{3}^{\prime} \rightarrow \text { Bew } \mathscr{2}}
$$

wird $\mathrm{zu}$ :

$$
\frac{\Gamma_{3, \Psi}^{\prime}, \Omega}{\Gamma_{3}^{\prime} \rightarrow \text { Bew } \mathfrak{S}^{\prime}}
$$

Man macht daraus : 


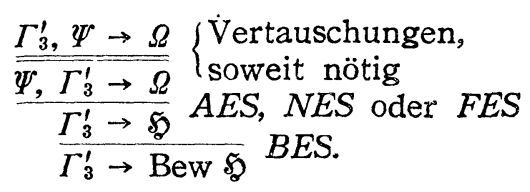

Im Falle $A E S$ ist klar, dass in AES die Variablenbedingung auf Grund der Umbenennung von freien Variablen erfüllt ist.

3.612.6. Die aus anderen Schlussfiguren, die die Formeln des Bundes enthalten, entstandenen Figuren sind sämtlich bereits Schlussfiguren, die von derselben Arten wie alten Schlussfiguren sind, aber die aus Vertauschungen, die die Formeln des Bundes als die Vertauschungsformeln haben, entstandenen Figuren sind die identischen Schlussfiguren. (Es ist klar, dass eine BES, die die Formeln des Bundes enthalten, ist die Endschlussfigur des Bundes, und dass in einer aus $A E S$ bzw. $E E A$ entstandene $A E S$ bzw. EEA die Variablenbedingung auf Grund der Umbenennung von freien Variablen erfüllt sind.)

Durch die obige Umwandlung haben wir eine $B L K$-Herleitung ohne Schnitt, die $\Gamma^{b} \rightarrow \Theta^{b}$ als die Endsequenz hat, und ihr Grad kleiner ist als der der alten $B L K$-Herleitung. Also ist $\Gamma^{b} \rightarrow \Theta^{b} B L J$-herleitbar nach Induktionsannahme.

Damit ist der Satz 3 vollständig bewiesen.

3.7. Nach den oben bewiesenen Sätzen 1, 2 und 3 gilt der folgende

Hauptsatz: Wenn das Verknüpfungszeichen "Bew " in $\Gamma$ und $\Theta$ nicht vorkommt, so ist

$$
\Gamma \rightarrow \Theta
$$

dann und nur dann $L J$-herleitbar, wenn

$$
\Gamma^{b} \rightarrow \Theta^{b}
$$

$B L K$-herleitbar ist.

\section{LITERATURVERZEICHNIS}

[1] G. Gentzen, Untersuchungen über das logische Schliessen. Math. Z. 39 (1935), S. 176210 und 405-431.

[2] S. Kuroda, Intuitionistische Untersuchungen der formalistischen Logik. Nagoya Math. J. 2 (1951), S. 35-47.

\section{Universität zu Tokyo}

